\title{
Usability, Acceptability, and Feasibility of Two Technology-Based Devices for Mental Health Screening in Perinatal Care: A Comparison of Web Versus App
}

\author{
Verónica Martínez-Borba ${ }^{1(\bowtie)}$, Carlos Suso-Ribera ${ }^{1}$, \\ and Jorge Osma ${ }^{2,3}$ \\ ${ }^{1}$ Universitat Jaume I, 12071 Castellón de la Plana, Spain \\ \{al189588,susor\}@uji.es \\ 2 Universidad de Zaragoza, 44003 Teruel, Spain \\ osma@unizar.es \\ ${ }^{3}$ Instituto de Investigación Sanitaria de Aragón, 50009 Zaragoza, Spain
}

\begin{abstract}
The use of Information and Communication Technologies (web pages and apps) in mental health has boosted. However, it is unknown which of these two devices can be better in terms of feasibility and acceptability. Our aim is to compare the feasibility, usability, and user satisfaction of two devices (web vs mobile application) of an online program for perinatal depression screening called HappyMom. In total, 348 and 175 perinatal women registered into HappyMom web and app version, respectively. The assessment protocol included different biopsychosocial evaluations (twice during pregnancy and thrice in the postpartum) and a satisfaction questionnaire. Results showed that a higher percentage of women in the web sample (27.3-51.1\%) responded to each assessment compared to the app sample (9.1-53.1\%). A smaller proportion of women in web sample never responded to any assessments. By contrast, the percentage of women who responded to all assessments was higher in app sample (longitudinal retention sample was $4.6 \%$ of web users and $9.1 \%$ of app users). In general, high satisfaction was found in both web and app users. Our result showed that online assessment methods are feasible and acceptable by perinatal women. However, dropout rates are a real problem that urge a solution that will be discussed further in the paper. Web and App devices present different advantages and limitations. The choice of one of them must be made taking into account the study's objective, the sample characteristics, and the dissemination possibilities.
\end{abstract}

Keywords: Information and Communication Technologies •

Dropouts $\cdot$ Satisfaction $\cdot$ Assessment $\cdot$ Perinatal women

\section{Introduction}

During the last decades, the use of Information and Communication Technologies (ICTs) in health (eHealth) have become widespread globally. The explosion of this technology is due to several factors. One of them is its low cost and high availability in 
the general population [1]. Other reasons include their advantages for the health service industry and research institutions. In the specific field of mental health, ICTs offer the possibility to overcome some barriers and limitations found in traditional face-to-face evaluations and interventions. Some of these advantages include accessibility and flexibility, reducing or eliminating the need to travel to the health center, anonymity, and the reduction of stigmatization associated with mental health consultation [2-5]. Not surprisingly, this has led to a great interest in the use of ICTs for mental and overall health-related issues in the past years [6-8].

Among the different ICTs that exist, the use of online platforms (i.e., web pages and mobile applications) has increased very quickly in health research and practice. As found in recent systematics reviews, apps and web pages have been used for delivering psychological treatment and routine monitoring in different populations with mental health problems, such as perinatal depression, chronic pain, social anxiety, eating disorders, borderline personality disorder, addictions, insomnia, stress, post-traumatic stress disorder, burnout, and suicidality, to name some examples [9-15].

In the specific field of perinatal depression, which is depressive symptoms that occur during pregnancy and up to 12 months postpartum [16], different web and appbased programs exist. Some of these screening, prevention, and treatment programs are web-based [17-21] while other are app-based [22-25]. Despite the wide use of these two online platforms, it is still unknown whether one of these two devices actually performs better in terms of feasibility (i.e., response rate), acceptability and usability (i.e., user satisfaction). Considering the widespread integration of smartphones into the daily life of individuals, mobile applications would be expected to present some advantages compared to web pages. For instance, mobile applications offer the possibility to conduct ecological momentary assessments (EMA), which involves repeated data collection (thoughts, behaviors and symptoms) in real time and in the natural environments of the individual [26, 27]. Additionally, smartphones allow collecting relevant antecedent information to the experience (i.e., localization or number of phone calls and SMS received). Consistent with this idea, a recent study showed that women who used a web-based treatment for perinatal depression would like the program to be available for its use on smartphones [3].

Although repeated assessment via ICTs might have important advantages, some possible limitations of this methodology should also be acknowledged. When assessments are made several times a day for a long period of time, dropout rates tend to occur [28], especially in self-administered online interventions [29]. Studies reporting attrition rates have found dropouts up to $50 \%$ in online treatment for depression and anxiety [30], showing that sample retention is one of the main concerns for studies using eHealth for monitoring and treatment [31]. In an effort to improve eHealth adherence, some authors have suggested that the psychological experience with the platform is important to determine usage and adherence to interventions [32], so acceptability and usability are key elements in this study.

In sum, the aim of this study was to compare the feasibility, usability, and user satisfaction of two devices (web vs mobile application) of an online program for perinatal depression screening called HappyMom (HM). We expect that feasibility, as measured by response rates and dropouts, as well as usability and acceptability will be 
better for mobile applications because this platform is more suited to be used in smartphones than the web version of Happymom.

\section{Method}

\subsection{Participants}

The sample was composed of 523 perinatal women who registered into HM. Of these, 348 women registered into the web version (HM-Web) and 175 participants used the app version (HM-App). Longitudinal assessments were carried out entirely with HM. Two evaluations were made during pregnancy (weeks 16-24 and 30-36 of gestation) and three in the postpartum (weeks 2, 4, and 12 after delivery). The assessment points were the same for both devices. The study was conducted between 2012 and 2015.

\subsection{Procedure}

The ethical committees of the Hospital Universitario La Plana de Villarreal and the Gobierno de Aragón approved the present study and its procedures.

$\mathrm{HM}$ is designed to evaluate mental well-being during perinatal period. The platform is composed by two devices: HM-Web and HM-App. A different procedure was followed depending on the device employed to better fit the characteristics of each device and to facilitate recruitment:

HM-Web. Participant recruitment was carried out at the health collaborating centers. Midwives offered the codes to pregnant women who met inclusion criteria. Adult women ( $>18$ years) between week 16 and 36 of gestation were invited to participate if they had internet access (at home or on the phone). Exclusion criteria included not being able to read or answer to the questions in Spanish. Women who voluntarily agreed to participate registered into the HM-Web and responded to different biopsychosocial assessments during pregnancy and the postpartum. Women were contacted via e-mail to complete each assessment at the required gestational stage (i.e., two assessments during pregnancy and three after delivery). Women who finished the evaluations received feedback about their mental well-being and those with depressive symptoms received a recommendation to consult their public health care service.

HM-App. Dissemination campaigns for recruitment were carried out via press, posters, e-mail, radio, and television. The link to download the app was posted in these social media. The HM-App was available for free download at Play Store (Android system). No healthcare professional support is integrated into the app. No code access was needed, so women who were interested in participating only had to download the HM-App and start using it. Given the free download character of the application, the age variable could not be controlled as an exclusion criteria. Inclusion criteria were being between week 16 and 36 of gestation at the time of download and being able to read and answer to the questions in Spanish. Women who agreed to participate registered into the HM-App to complete the biopsychosocial assessments during pregnancy and the postpartum. Once the women had registered into the HM-App, the app 
sent reminders to complete each biopsychosocial assessment at the required gestational stage. At the end of the evaluations, women received feedback about the mental wellbeing. When depressive symptoms were present, they were recommended to consult their public health care service.

\subsection{Measures}

The distribution of questionnaires administered according to the perinatal period and the device employed (HM-Web/HM-App) is shown in Table 1.

Questionnaires could be grouped into blocks according to their biological (obstetric data), psychological (emotional disorders, personality, affect, self-esteem, emotion regulation, quality of life, and satisfaction with life), or social (social adjustment, perceived social support, marital adjustment, stressful events, and familism) characteristics. In the postpartum, when the last biopsychosocial assessment was completed, all HM users responded to satisfaction questions.

The biopsychosocial measures changed between HM-App and HM-Web because of the limitations inherent to the use of smaller devices such as smartphones, so the app assessment was shorter. For that reason, the questionnaires in the HM-Web were divided in three blocks so the users could stop answering the questionnaires at any time and continuing answering where possible. In addition, some questionnaires (EPQ-R, STAI-T, ERQ, CAE, QLI and SRSS $)^{1}$ were administered only during pregnancy.

Table 1. Measures according to the perinatal period and device employed.

\begin{tabular}{|c|c|c|c|c|c|}
\hline \multirow[t]{3}{*}{ Questionnaires } & \multicolumn{5}{|c|}{ Perinatal period } \\
\hline & \multicolumn{2}{|l|}{ Pregnancy } & \multicolumn{3}{|c|}{ Postpartum } \\
\hline & Weeks $16-24$ & Weeks 30-36 & Week 2 & Week 4 & Week 12 \\
\hline \multirow[t]{2}{*}{ Register } & $1 \mathrm{Web}$ & & & & \\
\hline & 1 App & & & & \\
\hline \multirow[t]{2}{*}{ Sociodemographic } & $1 \mathrm{Web}$ & & & & \\
\hline & 1 App & & & & \\
\hline \multirow[t]{2}{*}{ Obstetric } & $1 \mathrm{Web}$ & $1 \mathrm{Web}$ & $1 \mathrm{Web}$ & $1 \mathrm{Web}$ & $1 \mathrm{Web}$ \\
\hline & $1 \mathrm{App}$ & $1 \mathrm{App}$ & $1 \mathrm{App}$ & $1 \mathrm{App}$ & $1 \mathrm{App}$ \\
\hline \multirow[t]{2}{*}{ Depressive symptoms [33-37] } & $3 \mathrm{Web}$ & $3 \mathrm{Web}$ & $3 \mathrm{Web}$ & 3 Web & $3 \mathrm{Web}$ \\
\hline & 1 App & $1 \mathrm{App}$ & $1 \mathrm{App}$ & $1 \mathrm{App}$ & $1 \mathrm{App}$ \\
\hline \multirow[t]{2}{*}{ Psychological factors [38-56] } & $10 \mathrm{Web}$ & $10 \mathrm{Web}$ & 5 web & 5 web & $5 \mathrm{Web}$ \\
\hline & 3 App & 3 App & 3 App & 3 App & 3 App \\
\hline \multirow[t]{2}{*}{ Social factors [57-65] } & $3 \mathrm{Web}$ & $3 \mathrm{Web}$ & $2 \mathrm{Web}$ & $2 \mathrm{Web}$ & $2 \mathrm{Web}$ \\
\hline & 2 App & 2 App & 2 App & 2 App & 2 App \\
\hline \multirow[t]{2}{*}{ Satisfaction } & & & & & $1 \mathrm{Web}$ \\
\hline & & & & & 1 App \\
\hline
\end{tabular}

${ }^{1}$ EPQ-R: Eysenck Personality Questionnaire Revised; STAI-T: State-Trait Anxiety Inventory (trait subscale); ERQ: Emotion Regulation Questionnaire; CAE: Cuestionario de Afrontamiento del Estrés; QLI: Quality of Life Index; SRSS: Social Readjustement Rating Scale. 


\subsection{Data Analysis}

First, we conducted a descriptive analysis of the sample. Then, we analyzed dropout rates (proportion of women who completed each assessment in relation to women who were registered into the program). Finally, we explored women's usability reports and satisfaction with HM.

\section{Results}

\subsection{Demographic Characteristics}

The HM-App sample comprised 175 women between 16 and 44 years of age (mean $=28.96 ; S D=6.34)$. The majority of them $(78.9 \%)$ were Spanish, $64 \%$ had completed secondary education studies or higher, and $76 \%$ of them were in a relationship at the time of the first assessment. With regards to the HM-Web, 348 women registered to this platform, but only 151 of them provided demographic information. Their age ranged from 20 to 42 years (mean $=32.77 ; S D=4.48$ ). Almost all women (92.1\%) were Spanish and the majority of them (78.8\%) had completed secondary education studies or higher and were in a relationship (76.8\%). Statistical differences between app and web users were found for age $(t=6.17 ; p<.001 ; 95 \% \mathrm{CI}=2.60$, 5.02), nationality $\left(\chi^{2}=11.05 ; p<.001 ; 95 \% \mathrm{CI}=5.56,20.62\right)$, and educational level $\left(\chi^{2}=8.57 ; p=.003 ; 95 \% \mathrm{CI}=4.94,24.13\right)$. Women who used the web were older, they had a higher educational level, and were more likely to be Spanish. There were no statistical differences in marital status $\left(\chi^{2}=0.03 ; p=.866 ; 95 \% \mathrm{CI}=-8.51,9.91\right)$.

\subsection{Dropout Rates}

HM-Web. Out of the 348 women who registered into HM-Web, 102 (29.3\%) did not complete any evaluation. During pregnancy, the percentage of women who completed all the questionnaires was $32.2 \%$ in the first evaluation and $51.1 \%$ in second assessment. In the postpartum, the proportion of women who completed all the questionnaires during the first, the second, or the third postpartum assessments were $38.2 \%$, $27.3 \%$, and $28.7 \%$, respectively. Only 16 women $(4.6 \%)$ responded to all the assessments.

HM-App. Out the 175 women who downloaded HM-App and registered into the program, $82(46.9 \%)$ did not complete any assessment. During pregnancy, the proportion of women who completed the first and the second evaluation was $53.1 \%$ and $21.7 \%$, respectively. In the postpartum, the percentage of women who completed the first, the second, or the third assessments was $13.7 \%, 10.9 \%$, and $9.1 \%$, respectively. Again, only 16 women $(9.1 \%)$ responded to all the evaluations.

As shown in Table 2, statistical differences in response rates between HM-App and HM-Web were found for all assessment points. Compared to HM-App users, more women registered into HM-Web and a larger percentage of women completed the assessments (i.e., second assessment during pregnancy and all of the postpartum 
evaluations) in the HM-Web. The proportion of women who completed all five assessments was higher in the HM-App, but the proportion of women who did not complete any evaluation was also higher in HM-App.

Table 2. Response rates according to the evaluation time and device used.

\begin{tabular}{l|l|l|l}
\hline \multirow{2}{*}{ Assessment } & \multicolumn{2}{|l|}{ Respondents $n(\%)$} & \multirow{2}{*}{ Chi-square test $(p, 95 \%$ CI $)$} \\
\cline { 2 - 3 } & HM-Web & HM-App & \\
\hline Registration & 348 & 175 & \\
\hline PRE 1 & $112(32.2)$ & $93(53.1)$ & $21.30(p<.001 ; 11.94$ to 29.53) \\
\hline PRE 2 & $178(51.1)$ & $38(21.7)$ & $41.45(p<.001 ; 20.91$ to 36.95$)$ \\
\hline POST 1 & $114(32.8)$ & $24(13.7)$ & $21.82(p<.001 ; 11.56$ to 25.78$)$ \\
\hline POST 2 & $95(27.3)$ & $19(10.9)$ & $18.33(p<.001 ; 9.36$ to 22.61$)$ \\
\hline POST 3 & $100(28.7)$ & $16(9.1)$ & $25.90(p<.001 ; 12.73$ to 25.63$)$ \\
\hline All assessments & $16(4.6)$ & $16(9.1)$ & $4.10(p=.043 ; 0.11$ to 9.97$)$ \\
\hline Any evaluation & $102(29.3)$ & $82(46.9)$ & $15.78(p<.001 ; 8.80$ to 26.26$)$ \\
\hline Satisfaction & $16(4.6)$ & $16(9.1)$ & $4.10(p=.043 ; 0.11$ to 9.97$)$ \\
\hline
\end{tabular}

\subsection{User's Satisfaction}

We registered 99 HM-Web satisfaction responses and 16 responses from HM-App. However, since only 16 of the 99 women using HM-Web completed the five assessment points, the analyses will be made both when considering the women who none completed all longitudinal measures ( $n=16$ for HM-Web and $n=16$ for HM-App) and the web non-completers ( $n=99$ for HM-Web and $n=16$ for HM-App). There was no difference in satisfaction when considering non-completers and completers for web users.

As shown in Table 3, all women considered studying perinatal well-being important. General satisfaction with HM was high for both, HM-Web and HM-App. The majority of women were satisfied with the registration process and indicated that the questions and instructions were easy to understand. Only $12.2 \%$ of web users reported difficulties when using the device. Despite the longitudinal character of the study and the number of questionnaires included, most women did not consider the time they devoted to answering the questions to be excessive ( $86.9 \%$ in the HM-Web sample and 93.8\% in the HM-App sample) and did not perceive their daily life had been altered (87.5\% in the HM-Web sample and $100 \%$ in the HM-App sample). Half of the women (50\% in HM-Web sample and $43.7 \%$ in HM-App sample) considered that the device was reliable for the assessment of mental well-being and about three fourths of women trusted the information received by HM.

The results regarding the perceived impact of HM in the mental well-being of participants and the future intention of use and willingness to recommend the platform to others revealed that one fourth of the sample perceived an improvement in their emotional well-being after using the platform. Additionally, between $50-75 \%$ of women (depending on the platform used) would use the application in a future pregnancy, while $62.5-75.8 \%$ of participants would recommend it to other women. 
Significant statistical differences were found in the perceived usefulness of the reminders to complete the assessments during the five evaluation points. A greater percentage of women in the HM-Web sample valued the reminders to complete the assessments positively compared to women in the HM-App sample. No other significant statistical differences were found in terms of user's satisfaction when comparing app and web users.

Table 3. HappyMom satisfaction according to device used.

\begin{tabular}{|c|c|c|c|}
\hline & \multicolumn{2}{|c|}{$\begin{array}{c}\text { Affirmative responses } \\
(\%)\end{array}$} & \multirow[t]{2}{*}{ Chi-square test $(p, 95 \% \mathrm{CI})$} \\
\hline & $\begin{array}{l}\text { HM- } \\
\text { Web* }\end{array}$ & $\begin{array}{l}\text { HM- } \\
\text { App }\end{array}$ & \\
\hline \multirow[t]{2}{*}{ Ease of registration } & 100 & 93.7 & $1.01(p=.316,-13.90$ to 28.29$)$ \\
\hline & 92 & & $0.05(p=.814,-20.74$ to 10.44$)$ \\
\hline \multirow{2}{*}{$\begin{array}{l}\text { Enough information about } \\
\text { HappyMom }\end{array}$} & 62.5 & 56.2 & $0.13(p=.721,-25.26$ to 36.19$)$ \\
\hline & 77.8 & & $3.38(p=.066,-0.99$ to 45.72$)$ \\
\hline \multirow[t]{2}{*}{ Ease of understand instructions } & 100 & 100 & \\
\hline & 97 & & $0.49(p=.486,-16.48$ to 8.48$)$ \\
\hline \multirow[t]{2}{*}{ Reminders utility } & 100 & 68.7 & $5.75(\boldsymbol{p}=\mathbf{. 0 1 6}, 5.45$ to 55.63$)$ \\
\hline & 95 & & $11.94(\boldsymbol{p}<.001,8.10$ to 50.81$)$ \\
\hline \multirow[t]{2}{*}{ Ease of web/app use } & 93.8 & 100 & $0.99(p=.320,-13.84$ to 28.25$)$ \\
\hline & 91.9 & & $1.38(p=.240,-11.67$ to 15.15$)$ \\
\hline \multirow[t]{2}{*}{ Ease of understand questions } & 87.5 & 100 & $2.06(p=.151,-8.87$ to 36.01$)$ \\
\hline & 84.9 & & $2.75(p=.097,-5.11$ to 23.43$)$ \\
\hline \multirow[t]{2}{*}{ Ease of respond questions } & 81.2 & 87.5 & $0.23(p=.629,-20.19$ to 32.18$)$ \\
\hline & 81.8 & & $0.31(p=.579,-18.67$ to 18.25$)$ \\
\hline \multirow[t]{2}{*}{ Difficulties with web/app } & 0 & 0 & \\
\hline & 12.2 & & $2.16(p=.142,-7.83$ to 20.09$)$ \\
\hline \multirow[t]{2}{*}{ Excessive time } & 0 & 6.2 & $0.94(p=.331-13.98$ to 28.16$)$ \\
\hline & 13.1 & & $0.61(p=.435,-15.79$ to 16.42$)$ \\
\hline \multirow{2}{*}{$\begin{array}{l}\text { Importance of studying perina- } \\
\text { tal mental well-being }\end{array}$} & 100 & 100 & \\
\hline & 100 & & \\
\hline \multirow[t]{2}{*}{ Reliability of HappyMom } & 50 & 43.7 & $0.12(p=.725,-25.58$ to 36.47$)$ \\
\hline & 67.7 & & $3.43(p=.064,-1.04$ to 46.28$)$ \\
\hline \multirow{2}{*}{$\begin{array}{l}\text { Confidence in the feedback } \\
\text { received }\end{array}$} & 75 & 68.7 & $0.15(p=.697,-23.58$ to 34.80$)$ \\
\hline & 82.8 & & $1.75(p=.186,-5.05$ to 39.21$)$ \\
\hline \multirow{2}{*}{$\begin{array}{l}\text { Conflict in the routine due to } \\
\text { the use of the device }\end{array}$} & 12.5 & 0 & $2.02(p=.155,-9.01$ to 35.92$)$ \\
\hline & 11.1 & & $1.91(p=.167,-9.01$ to 18.70$)$ \\
\hline \multirow{2}{*}{$\begin{array}{l}\text { Positive influence of the device } \\
\text { on well-being }\end{array}$} & 18.8 & 25 & $0.17(p=.676,-22.23$ to 33.56$)$ \\
\hline & 31.3 & & $0.26(p=.613,-19.56$ to 24.01$)$ \\
\hline \multirow[t]{2}{*}{ Future use of HappyMom } & 50 & 75 & $2.07(p=.151,-7.93$ to 51.52$)$ \\
\hline & 74.7 & & $0.01(p=.980,-25.33$ to 17.83$)$ \\
\hline \multirow[t]{2}{*}{ HappyMom recommendation } & 62.5 & 62.5 & \\
\hline & 75.8 & & $1.25(p=.263,-7.87$ to 38.27$)$ \\
\hline
\end{tabular}

*Note: shaded values corresponded to all web user's $(\mathrm{n}=99)$, web white values correspond to non-completers web user's ( $\mathrm{n}=16)$. 


\section{Discussion}

In recent year, an increasing number of eHealth programs, especially those based on web applications and smartphone applications, have emerged as an alternative to traditional face-to-face protocols as a result of the advantages of ICTs for health research and clinical purposes [9-15]. In relation to this literature on eHealth, some authors have highlighted the need to include participant engagement (i.e., acceptability and usability of the technology-based program) and retention rates as an key factors prior to the final implementation of ICT in routine practice [66]. In the field of perinatal depression, some studies have already reported encouraging acceptability and feasibility (i.e., dropout rates) results of eHealth, mostly after using web-based, online interventions $[3,18,67,68]$. However, to the best of our knowledge no study had previously compared the usability, satisfaction, and feasibility of the two most popular forms of online applications, namely web-based and smartphone applications. Thus, the aim of this study was to compare the feasibility, acceptability, and user satisfaction of two devices (web vs mobile application) of a perinatal mental health screening program. We hypothesized that feasibility (response rates and dropouts) and acceptability and satisfaction would be higher for the mobile application because of the widespread use of smartphone applications.

Regarding feasibility, our hypothesis was mostly not confirmed by our findings. Contrary to our predictions, a higher proportion of women did not complete any evaluation in the HM-App and response rates during pregnancy and the postpartum were consistently higher across almost all assessment points in the HM-Web group. Additionally, the proportion of women who did not complete any assessment after registration was lower for the HM-Web (29.3\%) compared to the HM-App (46.9\%). Only retention occurred as expected, in the sense that a greater percentage of women using the HM-App completed all assessments.

Several reasons might explain these aforementioned findings regarding feasibility. For instance, it is possible that higher response rates in the HM-Web group are due to differences in the sample recruitment method. Recruitment in the HM-Web was conducted with the collaboration of health care professionals who delivered the web access codes to women, mainly midwives. By contract, the HM-App was available for free download, with no health care professional collaboration for its dissemination. It is possible that women were more motivated to use HM at least once when they perceived that health care professionals were involved in the program. As suggested in a recent study [69], it is possible that some women do not trust information from health-related websites and apps, so it is necessary to involve health care professionals in the dissemination of ICT in perinatal care. Moreover, disseminating campaigns should be supported by public and private health services, promoting routine assessments of emotional well-being during perinatal period. We tried to minimize this risk by offering both groups a reward for participating (i.e., feedback about their well-being), but maybe the effect of the institution was still larger. Another possible explanation for these findings is anonymity. The stigma of mental illness is a frequent issue during the perinatal period [70] and some perinatal women report confidentiality or privacy concerns regarding online programs for well-being [71]. In the HM-Web, women did 
not need to download any application that would be visible in the phone. In the HMWeb, women simply needed to access the website (which can be done from any device) and complete the evaluations. While this remains speculative at this stage, it is possible that this procedure was perceived as more anonymous than downloading and installing an app that would be visible in the phone, thus leading to an increased number of responses in the HM-Web.

While acknowledging the previous findings in favor of the HM-Web, it is interesting that the proportion of women who completed all five assessments was higher in the HM-App. This indicates, in line with our hypothesis, a higher fidelity to the app platform. Again, while the following conclusions are merely speculative, it is possible that the easier accessibility and widespread use of smartphones apps make these platforms more attractive for some people when it comes to repeated measurement. Indeed, there is previous literature to suggest that some women are interested in having web-based interventions adapted to smartphone apps [3], so this platform appears to be an attractive option for a number of users and should be considered when repeated measurement is planned. It is important to note, however, that retention rates were low both in the HM-Web and the HM-App (4.5\% and 9.1\%, respectively), which confirms that longitudinal studies result in high dropout rates [28], especially when a large number of questionnaires is administered like in the present study [29]. The results suggest that long assessments with a wide number of measures might be counterproductive, so strategies like questionnaire reduction or even single-item use for the measurement of constructs should be considered [8]. This could be combined with the inclusion of motivating practices, such as gamification or benefits for participating (i.e., access to an online chat with a mental health professional).

Regarding women's satisfaction with HM, we anticipated that the satisfaction with the HM-App would be higher when compared to the HM-Web. Our results do not support this hypothesis and indicated a good satisfaction for both HM versions. Previous research $[3,18,72]$ has also showed that online platforms have high acceptability by perinatal women. HM was not an exception and both devices were perceived as easy to use and very well accepted, including no technical difficulties, straightforward questions, adequate response time, minimal impact on the individual's routine, and trust in the feedback received about emotional well-being. The only difference in HM satisfaction refers to the utility of reminders. Specifically, women who used the web were more grateful that e-mails were sent to them reminding them to respond to the evaluations. What these results suggest is that reminders are less necessary for the app version of the program, arguably because of the less extended assessment protocol and the frequent use of smartphones in our daily living.

Despite the general satisfaction with HM, its perceived impact in the mental wellbeing of participants and the future intention to use the platform and to recommend it to others was modest. As suggested by previous studies, screening programs are not sufficient for perinatal depression treatment and prevention [73, 74], so it is not surprising that a quarter of women in our sample did not perceive any improvement in their emotional well-being after using HM, which might explain their low interest in using the platform in the future and recommending it to others. Future screening programs should not only provide feedback about emotional status, but also offer other resources valued for perinatal women such as information, tips or the possibility of an 
online treatment via ICT if necessary. While these findings are important and suggest a need to improve screening programs for perinatal women using technology, it should be noted that all women in our sample considered the study of perinatal well-being a fundamental goal. Considering that women have considered HM and other online platforms for perinatal care to be easy and acceptable tools for the assessment of their well-being, future health campaigns should focus on implementing ICT protocols to perinatal mental health assessment and treatment in routine care.

Our study certainly has some limitations. Participant recruitment was different in the HM-Web and the HM-App, so that only the web was recommended by health care professionals. As discussed above, this could affect the final sample obtained in the web and the app (both numbers and their motivation to participate). Additionally, because of HM characteristics, only women who were able to understand and answer questions in Spanish were able to participate in the study, thus limiting the generalization of results to all perinatal women. Related to this, the majority of women were from Spain, so nationality comparison was not possible. It is possible that feasibility and satisfaction results of ICTs programs for perinatal mental health are higher in countries were the use of eHealth is more frequent (i.e., United States of America or United Kingdom). It is also important to note that our satisfaction results are based on the opinion of women who completed all evaluations, as women who did not respond to the last biopsychosocial assessment could not complete the satisfaction survey. In future research, we should include and better comprehend the opinions of participant who left the study, but this was not possible in the present investigation because of the characteristics of the study (i.e., the phone number or email address of women using the app were not collected to increase the sense of anonymity). Finally, there were some differences in the assessment protocol in the web and in the app, so differences in dropout rates should be considered with caution.

To sum up, our results show that online platforms (web-based and app-based) can be reliable and acceptable methods to deliver mental health assessment during the perinatal period. User's satisfaction was good for both devices, especially regarding ease of use, time devoted to the platform, and interference with daily life. The HMWeb obtained overall better results in terms of feasibility (i.e., number of registrations and percentage of women who completed each assessment), which suggests that perinatal mental health evaluations can be carried out successfully via web-pages. While the following is merely speculative, our results might also suggest that response rates will be higher if health care professionals are involved in dissemination. Interestingly, though, retention rates were in favor of the HM-App, so mobile applications might be suitable tools for longitudinal assessment. While acknowledging the previous feasibility findings, it is important to note that response rates were generally low, so strategies when performing repeated assessment using technology (i.e., gamification and benefits of participation) should be implemented in future research using technology for perinatal care. 


\section{References}

1. International Telecommunication Union: ICT Facts and Figures (2017). https://www.itu.int/ en/ITU-D/Statistics/Pages/facts/default.aspx

2. Barrera, A.Z., Kelman, A.R., Muñoz, R.F.: Keywords to recruit Spanish- and Englishspeaking participants: evidence from an online postpartum depression randomized controlled trial. J. Med. Internet Res. 16, e6 (2014)

3. Haga, S.M., Drozd, F., Brendryen, H., Slinning, K.: Mamma mia: a feasibility study of a web-based intervention to reduce the risk of postpartum depression and enhance subjective well-being. JMIR Res. Protoc. 2, e29 (2013)

4. Kim, H., Bracha, Y., Tipnis, A.: Automated depression screening in disadvantaged pregnant women in an urban obstetric clinic. Arch. Womens Ment. Health 10, 163-169 (2007)

5. Donker, T., Cuijpers, P., Stanley, D., Danaher, B.: The future of perinatal depression identification can information and communication technology optimize effectiveness? (2015)

6. Eurostat: Internet use. Individuals using the Internet for seeking health-related information. http://ec.europa.eu/eurostat/tgm/table.do?tab=table\&plugin=1\&language=en\&pcode= tin00101

7. Osma, J., Barrera, A.Z., Ramphos, E.: Are pregnant and postpartum women interested in health-related apps? Implications for the prevention of perinatal depression. Cyberpsychol. Behav. Soc. Netw. 19, 412-415 (2016)

8. Suso-Ribera, C., Castilla, D., Zaragozá, I., Ribera-Canudas, M.V., Botella, C., GarcíaPalacios, A.: Validity, reliability, feasibility, and usefulness of pain monitor, a multidimensional smartphone app for daily monitoring of adults with heterogeneous chronic pain. Clin. J. Pain 34, 900-908 (2018)

9. Ashford, M.T., Olander, E.K., Ayers, S.: Computer- or web-based interventions for perinatal mental health: a systematic review. J. Affect. Disord. 197, 134-146 (2016)

10. Bakker, D., Kazantzis, N., Rickwood, D., Rickard, N.: Mental health smartphone apps: review and evidence-based recommendations for future developments. JMIR Ment. Health 3, 67 (2016)

11. Capon, H., Hall, W., Fry, C., Carter, A.: Realising the technological promise of smartphones in addiction research and treatment: an ethical review. Int. J. Drug Policy 36, 47-57 (2016)

12. Martínez-Borba, V., Suso-ribera, C., Osma, J.: The use of information and communication technologies in perinatal depression screening. Cyberpsychol. Behav. Soc. Netw. 21, 741752 (2018)

13. Pospos, S., et al.: Web-based tools and mobile applications to mitigate burnout, depression, and suicidality among healthcare students and professionals: a systematic review. Acad. Psychiatry 42, 109-120 (2017)

14. Rathbone, A., Clarry, L., Prescott, J.: Assessing the efficacy of mobile health apps using the basic principles of cognitive behavioral therapy: systematic review. J. Med. Internet Res. 19, e399 (2017)

15. Richards, D., Richardson, T.: Computer-based psychological treatments for depression: a systematic review and meta-analysis. Clin. Psychol. Rev. 32, 329-342 (2012)

16. Hamel, C., et al.: Screening for depression in women during pregnancy or the first year postpartum and in the general adult population: a protocol for two systematic reviews to update a guideline of the Canadian Task Force on Preventive Health Care. Syst. Rev. 8, 1-13 (2019)

17. Barrera, A.Z., Wickham, R.E., Muñoz, R.F.: Online prevention of postpartum depression for Spanish- and English-speaking pregnant women: a pilot randomized controlled trial. Internet Interv. 2, 257-265 (2015) 
18. Danaher, B.G., et al.: MomMoodBooster web-based intervention for postpartum depression: feasibility trial results. J. Med. Internet Res. 15, e242 (2013)

19. Jones, B.A., Griffiths, K.M., Christensen, H., Ellwood, D., Bennett, K., Bennett, A.: Online cognitive behaviour training for the prevention of postnatal depression in at-risk mothers: a randomised controlled trial protocol. BMC Psychiatry 13, 265 (2013)

20. Le, H.-N., Perry, D.F., Sheng, X.: Using the internet to screen for postpartum depression. Matern. Child Health J. 13, 213-221 (2009)

21. O'Mahen, H.A., et al.: Internet-based behavioral activation-treatment for postnatal depression (Netmums): a randomized controlled trial. J. Affect. Disord. 150, 814-822 (2013)

22. Faherty, L.J., Hantsoo, L., Appleby, D., Sammel, M.D., Bennett, I.M., Wiebe, D.J.: Movement patterns in women at risk for perinatal depression: use of a mood-monitoring mobile application in pregnancy. J. Am. Med. Inform. Assoc. 24, 746-753 (2017)

23. Jimenez-Serrano, S., Tortajada, S., Miguel Garcia-Gomez, J., Jiménez-Serrano, S., Tortajada, S., García-Gómez, J.M.: A mobile health application to predict postpartum depression based on machine learning. Telemed. E-Health 21, 567-574 (2015)

24. Belisario, J.S.M., et al.: A bespoke mobile application for the longitudinal assessment of depression and mood during pregnancy: protocol of a feasibility study. BMJ Open 7, e014469 (2017)

25. Tsai, A.C., et al.: Antenatal depression case finding by community health workers in South Africa: feasibility of a mobile phone application. Arch. Womens Ment. Health. 17, 423-431 (2014)

26. Demirci, J., Bogen, D.: Feasibility and acceptability of a mobile app in an ecological momentary assessment of early breastfeeding. Matern. Child Nutr. 13, 1-18 (2017)

27. Timmer, B.H.B., Hickson, L., Launer, S.: Ecological momentary assessment: Feasibility, construct validity, and future applications. Am. J. Audiol. 26, 436-442 (2017)

28. Young, A.F., Powers, J.R., Bell, S.L.: Attrition in longitudinal studies: who do you lose? Aust. N. Z. J. Public Health 30, 353-361 (2006)

29. Karyotaki, E., et al.: Predictors of treatment dropout in self-guided web-based interventions for depression: an 'individual patient data' meta-analysis. Psychol. Medici. 45, 2717-2726 (2015)

30. Christensen, H., Griffiths, K.M., Farrer, L.: Adherence in internet interventions for anxiety and depression. J. Med. Internet Res. 11, e13 (2009)

31. Becker, S., Miron-Shatz, T., Schumacher, N., Krocza, J., Diamantidis, C., Albrecht, U.-V.: mHealth 2.0: experiences, possibilities, and perspectives. JMIR mHealth uHealth 2, e24 (2014)

32. Feather, J.S., Howson, M., Ritchie, L., Carter, P.D., Parry, D.T., Koziol-McLain, J.: Evaluation methods for assessing users' psychological experiences of web-based psychosocial interventions: a systematic review. J. Med. Internet Res. 18, e181 (2016)

33. Beck, A., Steer, R., Brown, G.: Manual for the Beck Depression Inventory-II. Psychological Corporation, San Antonio (1996)

34. Sanz, J., Perdigón, A., Vázquez, C.: Adaptación española del Inventario para la Depresión de Beck-II (BDI-II): 2. Propiedades psicométricas en población general. Clin. Salud. 14, 249$280(2003)$

35. Cox, J.L., Holden, J.M., Sagovsky, R.: Detection of postnatal depression: development of the 10-item edinburgh postnatal depression scale. Br. J. Psychiatry 150, 782-786 (1987)

36. Garcia-Esteve, L., Ascaso, C., Ojuel, J., Navarro, P.: validation of the Edinburgh Postnatal Depression Scale (EPDS) in Spanish mothers. J. Affect. Disord. 75, 71-76 (2003)

37. First, M., Spitzer, R., Gibbon, M., Williams, J.: Entrevista Clínica estructurada para los trastornos del Eje II del DSM-IV, Versión Clínica. MASSON, Barcelona (1999) 
38. Eysenck, S.B.G., Eysenck, H.J., Barrett, P.: A revised version of the psychoticism scale. Pers. Individ. Diff. 6, 21-29 (1985)

39. Ortet, G., Ibáñez, M., Moro, M., Silva, F.: Cuestionario revisado de Personalidad de Eysenck: versiones completa (EPQ-R) y abreviada (EPQ-RS), Madrid (2001)

40. Brown, T.A., White, K.S., Forsyth, J.P., Barlow, D.H.: The structure of perceived emotional control: psychometric properties of a revised anxiety control questionnaire. Behav. Ther. 35, 75-99 (2004)

41. Osma, J., Barrada, J.R., Garciá-Palacios, A., Navarro-Haro, M., Aguilar, A.: Internal structure and clinical utility of the Anxiety Control Questionnaire-Revised (ACQ-R) Spanish version. Span. J. Psychol. 19, E63 (2016)

42. Sandín, B., Chorot, P., Lostao, L., Joiner, T.E., Santed, M.A., Valiente, R.M.: Escalas PANAS de afecto positivo y negativo: validacion factorial y convergencia transcultural. Psicothema 11, 37-51 (1999)

43. Watson, D., Clark, L.A., Tellegen, A.: Development and validation of brief measures of positive and negative affect: the PANAS scales. J. Pers. Soc. Psychol. 54, 1063 (1988)

44. Morejón, A.J.V., García-Bóveda, R.J., Jiménez, R.V.-M.: Escala de autoestima de Rosenberg: fiabilidad y validez en población clínica española. Apunt. Psicol. 22, 247-255 (2004)

45. Rosenberg, M.: Society and the Adolescent Self-image. Princeton University Press, Princeton (1965)

46. Spielberger, C., Cubero, N., Gorsuch, R., Lushene, R.: Cuestionario de ansiedad estadorasgo: manual, Madrid (1982)

47. Spielberger, C.D., Gorsuch, R.L., Lushene, R.E.: The State-Trait Anxiety Inventory. Consulting Psychologists Press Inc., Palo Alto (1970)

48. Echeburúa, E., de Corral, P., Fernandez-Montalvo, J.: Escala de inadaptación (EI): propiedades psicométricas en contextos clínicos. Anál. Modif. Conduct. 26, 325-340 (2000)

49. Rodríguez-Carvajal, R., Moreno-Jiménez, B., Garrosa, E.: Cuestionario de regulación emocional. Versión española. Autorizado por los autores de la versión original en inglés (Gross y John, 2003) (2006)

50. Gross, J., John, O.: Individual differences in two emotion regulation processes: implications for affect, relationships, and well-being. J. Pers. Soc. Psychol. 85, 348-362 (2003)

51. Sandín, B., Chorot, P.: Cuestionario de Afrontamiento del Estrés (CAE): Desarrollo y validación preliminar. Rev. Psicopatol. Psicol. Clín. 8, 39-53 (2003)

52. Mezzich, J., Ruipérez, M., Pérez, C., Yoon, G., Liu, J., Mahmud, S.: The Spanish version of the quality of life index: presentation and validation. J. Nerv. Ment. Dis. 188, 301-305 (2000)

53. Beck, A., Steer, R.: Manual for the Beck Anxiety Inventory. The Psychological Corporation, San Antonio (1993)

54. Beck, A., Steer, R.: Manual. BAI. Inventario de Ansiedad de Beck (Adaptación española de Sanz, J.). Pearson Educación, Madrid (2011)

55. Diener, E., Emmons, R., Larsen, R., Grifin, S.: The satisfaction with life scale. J. Pers. Assess. 49, 71-75 (1985)

56. Atienza, F.: Escala de Satisfacción con la vida. Psicothema 12, 314-319 (2000)

57. González de Rivera, J., Morera, A.: La valoración de sucesos vitales: adaptación española de la escala de Holmes y Rahe. Psiquis (Mexico) 4, 7-11 (1983)

58. Holmes, T.H., Rahe, R.H.: The social readjustment rating scale. J. Psychosom. Res. 11, 213 218 (1967)

59. Landeta, O., Calvete, E.: Adaptación y validación de la escala multidimensional de apoyo social percibido. Ansiedad Estrés 8, 173-182 (2002) 
60. Zimet, G.D., Dahlem, N.W., Zimet, S.G., Farley, G.K.: The multidimensional scale of perceived social support. J. Pers. Assess. 52, 30-41 (1988)

61. Carrobles, J.: Adaptación en población española de la Escala de Ajuste Marital de LockeWallace. In: Cáceres Carrasco, J. (ed.) Manual de Terapia de Pareja en Intervención en familias, pp. 105-106 (1989)

62. Locke, H.J., Wallace, K.M.: Short marital-adjustment and prediction tests: their reliability and validity. Marriage Fam. Living 21, 251-255 (1959)

63. Rodríguez-Muñoz, M.F., Vallejo Slocker, L., Olivares Crespo, M.E., Izquierdo Méndez, N., Soto, C., Le, H.-N.: Propiedades psicométricas del Postpartum Depression Predictors Iinventory-revised-versión prenatal en una muestra española de mujeres embarazadas. TT [Psychometric properties of postpartum depression predictors inventory-revised- prenatal version in a sample of Spanish pregnant women]. Rev. Esp. Salud. Publica 91, e1-e8 (2017)

64. Beck, C., Records, K., Rice, M.: Further development of the postpartum depression predictors inventory-revised. J. Obstet. Gynecol. Neonatal Nurs. 35, 735-745 (2006)

65. Villarreal, R., Blozis, S.A., Widaman, K.F.: Factorial invariance of a pan-hispanic familism scale. Hisp. J. Behav. Sci. 27, 409-425 (2005)

66. Lane, T.S., Armin, J., Gordon, J.S.: Online recruitment methods for web-based and mobile health studies: a review of the literature. J. Med. Internet Res. 17, e183 (2015)

67. Logsdon, M.C., Foltz, M.P., Stein, B., Usui, W., Josephson, A.: Adapting and testing telephone-based depression care management intervention for adolescent mothers. Arch. Womens Ment. Health 13, 307-317 (2010)

68. Baker-Ericzén, M.J., Connelly, C.D., Hazen, A.L., Dueñas, C., Landsverk, J.A., Horwitz, S. M.: A collaborative care telemedicine intervention to overcome treatment barriers for Latina women with depression during the perinatal period. Fam. Syst. Health 30, 224-240 (2012)

69. Fernández, M.: Herramienta metodológica para la evaluación de contenidos digitales para las gestantes. Interv. Eval. Matronas Hoy 3, 14-25 (2015)

70. Maloni, J.A., Przeworski, A., Damato, E.G.: Web recruitment and internet use and preferences reported by women with postpartum depression after pregnancy complications. Arch. Psychiatr. Nurs. 27, 90-95 (2013)

71. Hantsoo, L., Podcasy, J., Sammel, M., Epperson, C.N., Kim, D.R.: Pregnancy and the acceptability of computer-based versus traditional mental health treatments. J. Womens Health (Larchmt) 26, 1106-1113 (2017)

72. Kim, H.G., Geppert, J., Quan, T., Bracha, Y., Lupo, V., Cutts, D.B.: Screening for postpartum depression among low-income mothers using an interactive voice response system. Matern. Child Health J. 16, 921-928 (2012)

73. Miller, L., Shade, M., Vasireddy, V.: Beyond screening: assessment of perinatal depression in a perinatal care setting. Arch. Womens Ment. Health 12, 329-334 (2009)

74. Kingston, D., et al.: Study protocol for a randomized, controlled, superiority trial comparing the clinical and cost-effectiveness of integrated online mental health assessment-referral-care in pregnancy to usual prenatal care on prenatal and postnatal mental health and infant. Trials 15 (2014) 